\title{
Efficient split field FDTD analysis of third-order nonlinear materials in two-dimensionally periodic media
}

\author{
Jorge Francés ${ }^{\mathrm{a}}$, Sergio Bleda ${ }^{\mathrm{a}}$, Subhajit Bej ${ }^{\mathrm{b}}$, Jani Tervo ${ }^{\mathrm{b}}$, Víctor Navarro-Fuster ${ }^{\mathrm{a}}$, Sandra \\ Fenoll $^{\mathrm{a}}$, Francisco J. Martínez-Guardiola ${ }^{\mathrm{a}}$, and Cristian Neipp ${ }^{\mathrm{a}}$ \\ aDepartamento de Física, Ingeniería de Sistemas y Teoría de la Señal, Universidad de \\ Alicante,, San Vicente del Raspeig Drive, NW,Alicante, Ap. 99, E-3080, Spain \\ ${ }^{b}$ Institute of Photonics, University of Eastern Finland,P.O. Box 111, FI-80101 Joensuu, \\ Finland
}

\begin{abstract}
In this work the split-field finite-difference time-domain method (SF-FDTD) has been extended for the analysis of two-dimensionally periodic structures with third-order nonlinear media. The accuracy of the method is verified by comparisons with the nonlinear Fourier Modal Method (FMM). Once the formalism has been validated, examples of one- and two-dimensional nonlinear gratings are analysed. Regarding the $2 \mathrm{D}$ case, the shifting in resonant waveguides is corroborated. Here, not only the scalar Kerr effect is considered, the tensorial nature of the third-order nonlinear susceptibility is also included. The consideration of nonlinear materials in this kind of devices permits to design tunable devices such as variable band filters. However, the third-order nonlinear susceptibility is usually small and high intensities are needed in order to trigger the nonlinear effect. Here, a one-dimensional CBG is analysed in both linear and nonlinear regime and the shifting of the resonance peaks in both TE and TM are achieved numerically. The application of a numerical method based on the finitedifference time-domain method permits to analyse this issue from the time domain, thus bistability curves are also computed by means of the numerical method. These curves show how the nonlinear effect modifies the properties of the structure as a function of variable input pump field. When taking the nonlinear behaviour into account, the estimation of the electric field components becomes more challenging. In this paper, we present a set of acceleration strategies based on parallel software and hardware solutions.
\end{abstract}

Keywords: Manuscript format, template, SPIE Proceedings, LaTeX

\section{INTRODUCTION}

Photonics devices based on nonlinear optics have been extensively investigated in recent years due to the wide range of application areas such as communications and optical computing. ${ }^{1}$ More specifically, optical switches and modulators have been designed taking into account the nonlinear properties of various materials. ${ }^{2}$ One of the most interesting phenomena achievable in nonlinear materials is the all-optical control of electromagnetic waves, which allows all-optical signal processing. Its application to photonic crystals and periodic optical media in general has been extensively investigated in literature ${ }^{3}$ but currently numerical simulation, which is necessary in the design of sophisticated photonics devices, is very challenging. As a result, it is important to develop numerical methods that can easily and accurately simulate nonlinear optical process in complex periodic structures.

The Split-Field Finite-Difference Time-Domain (SF-FDTD) approach is a formulation of FDTD that is specially tailored to efficiently incorporate the periodicity in the algorithm (SF-FDTD). ${ }^{4-11}$ Namely, SF-FDTD provides a natural framework for simulating periodic optical media under oblique incidence by means of the application of the periodic boundary conditions (PBC). Simulating periodic structures with the standard FDTD approach implied to consider a non trivial number of periods in order to simulate accurately periodic structures. In SF-FDTD only one period is considered rather than the whole structure, which has obvious advantages as

Further author information: (Send correspondence to J. Francés.)

J. Francés.: E-mail: jfmonllor@ua.es, Telephone: +34 965903400 Ext: 1158

Optical Modelling and Design IV, edited by Frank Wyrowski, John T. Sheridan, Youri Meuret, Proc. of SPIE Vol. 9889, $988908 \cdot$ (c) 2016 SPIE · CCC code: 0277-786X/16/\$18 · doi: 10.1117/12.2227542 
(a)

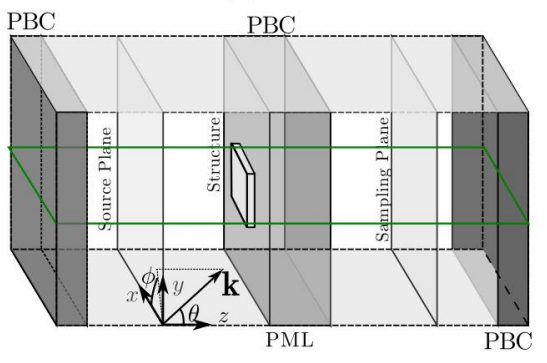

(b)

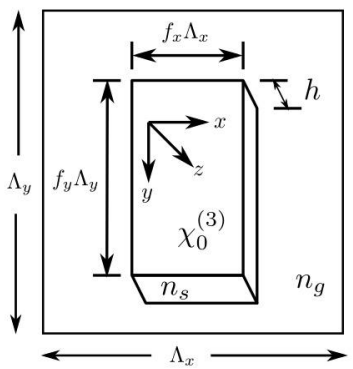

(c)

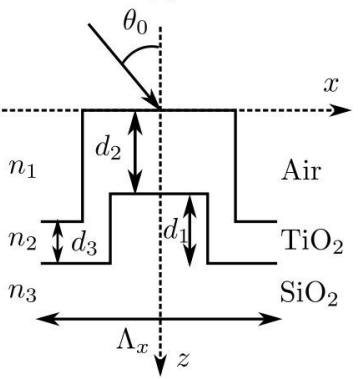

Figure 1. (a) General problem space for SF-FDTD simulations used in this paper. (b) Top view of the unit cell of a rectangular two-dimensially-periodic grating. (c) Cross-section of a coated binary grating.

far as numerical efficiency is concerned. However, this scheme implies complex notation and a double timeupdate of the electromagnetic field in each time step. This implies an increase of both the time and memory resources compared with the standard FDTD approach. Therefore, parallel strategies should be considered in order to accelerate the method if two-dimensionally periodic structures and thus three-dimensional problems are considered.

Although the classical FDTD formulation covers nonlinear media, ${ }^{12}$ the Split-Field approach must be thoroughly re-formulated in such cases. Recently, the SF-FDTD was extended for simulating second- and third-order nonlinear media for one-dimensionally periodic structures. ${ }^{13,14}$ In this paper, we introduce an approach allowing efficient simulation of two-dimensionally periodic structures, such as planar photonic crystals or crossed gratings. We validate our method by comparing the results with those obtained by iterative nonlinear Fourier modal method (FMM). ${ }^{15}$ Finally, shifting of diffraction efficiency curves is also corroborated by means of the analysis of three-dimensional binary gratings with pillars filled with nonlinear materials.

\section{THIRD-ORDER NONLINEAR SPLIT-FIELD FINITE-DIFFERENCES METHOD}

Fig. 1a shows the problem space for a typical SF-FDTD simulation of periodic media. We assume that the structure is periodic in $x$ and $y$ directions, and the incoming plane wave propagates predominantly in the $z$ direction, although the propagation angles $\theta$ between the wave vector $\mathbf{k}_{0}$ and the positive $z$ axis, and the azimuthal angle $\phi$ from the $z$ axis are not restricted. Both $z$ boundaries are terminated with Perfectly-Matched Layers (PMLs) that eliminate undesired reflections. ${ }^{7,8,16}$ The $x y$ boundaries are terminated with the Periodic Boundary Conditions (PBC). In order to accomplish this task successfully, the split field method uses a set of transformed fields that eliminate the phase difference between the two sides of the problem space.

Assuming non-magnetic, and non-conducting media, Maxwell's curl equations take the following form

$$
\nabla \times \mathbf{E}=-\mu_{0} \frac{\partial \mathbf{H}}{\partial t}, \quad \nabla \times \mathbf{H}=\frac{\partial \mathbf{D}}{\partial t},
$$

where the electric field is denoted by $\mathbf{E}$ whereas the magnetic field is $\mathbf{H}$. The vacuum permeability is $\mu_{0}$ and $\mathbf{D}$ is the flux density, which can be split to the linear and the nonlinear parts as

$$
\begin{gathered}
\mathbf{D}=\epsilon_{0} \epsilon_{r} \mathbf{E}+\mathbf{F}^{\mathrm{NL}}, \quad \mathbf{F}^{\mathrm{NL}}=\epsilon_{0} \sum_{j} \chi_{i j}^{\mathrm{eff}} E_{j}, \\
\chi_{i j}^{\mathrm{eff}}=A\left(\mathbf{E E}^{*}\right) \delta_{i j}+\frac{1}{2} B\left(E_{i} E_{j}^{*}+E_{i}^{*} E_{j}\right),
\end{gathered}
$$

where $A$ and $B$ and their relative amplitude depends on the nature of the physical process that produces the optical nonlinearity. ${ }^{1}$

In SF-FDTD,$^{7-10}$ we consider a transformation of the electric and magnetic fields, taking into account that the new variables implicitly contain the oblique field propagation. Firstly, the plane wave propagating vector 
(see Fig. 1a) is defined as $\mathbf{k}=k_{x} \hat{x}+k_{y} \hat{y}+k_{z} \hat{z}=\frac{\omega}{c}(\sin \theta \cos \phi \hat{x}+\sin \theta \sin \phi \hat{y}+\cos \theta \hat{z})$ where $\omega$ is the angular frequency and $c$ is speed of light in vacuum. Thus, the new split-field variables are

$$
\mathbf{P}=\mathbf{E} e^{j\left(k_{x} x+k_{y} y\right)}, \quad \mathbf{Q}=c \mu_{0} \mathbf{H} e^{j\left(k_{x} x+k_{y} y\right)},
$$

where $\mathbf{P}$ and $\mathbf{Q}$ are the transformed vectors in the phasor domain of the electric $\mathbf{E}$ and the magnetic $\mathbf{H}$ fields respectively.

Substituting the split-field components into Maxwell's equations, ${ }^{8,13}$ the basis for the SF-FDTD with thirdorder nonlinear polarization terms can be expressed as follows

$$
\nabla \times \mathbf{P}=-\frac{j \omega}{c} \mathbf{Q}-j \frac{\omega}{c} \mathbf{q} \mathbf{P}, \quad \nabla \times \mathbf{Q}=\frac{\epsilon_{r}}{c} j \omega \mathbf{P}+j \frac{\omega}{c} \sum_{i} \chi_{i j}^{\text {eff }} P_{j},
$$

where the matrix $\mathbf{q}$ is defined as

$$
\mathbf{q}=\frac{\omega}{c}\left[\begin{array}{ccc}
0 & 0 & -k_{y} \\
0 & 0 & k_{x} \\
k_{y} & -k_{x} & 0
\end{array}\right]
$$

The linear susceptibility term can be easily transformed into the split-field domain since $\mathbf{P} \mathbf{P}^{*}=\mathbf{E} \mathbf{E}^{*}=|\mathbf{E}|^{2}=\mathbf{I}$, thus

$$
\chi_{i j}^{\mathrm{eff}}=A \mathbf{I} \delta_{i j}+\frac{1}{2} B\left(P_{i} P_{j}^{*}+P_{i}^{*} P_{j}\right)
$$

We find that final update relations for the electromagnetic split-field domain the third-order nonlinear polarization terms can be derived from Eqs. (7)-(5)

$$
\begin{aligned}
P_{x} & =\frac{P_{x a}-\kappa\left[k_{y} Q_{z a}-k_{x} k_{y} P_{y}\right]-\kappa B\left[\Re\left\{P_{x} P_{y}^{*}\right\} P_{y}+\Re\left\{P_{x} P_{z}^{*}\right\} P_{z}\right]}{1+\kappa\left[k_{y}^{2}+\mathbf{I} A+I_{x} B\right]}, \\
P_{y} & =\frac{P_{y a}+\kappa\left[k_{x} Q_{z a}+k_{x} k_{y} P_{x}\right]-\kappa B\left[\Re\left\{P_{y} P_{x}^{*}\right\} P_{x}+\Re\left\{P_{y} P_{z}^{*}\right\} P_{z}\right]}{1+\kappa\left[k_{x}^{2}+\mathbf{I} A+I_{y} B\right]}, \\
P_{z} & =\frac{P_{z a}-\kappa\left[k_{y} Q_{x a}-k_{x} Q_{y a}\right]-\kappa B\left[\Re\left\{P_{z} P_{x}^{*}\right\} P_{x}+\Re\left\{P_{z} P_{y}^{*}\right\} P_{y}\right]}{1-\kappa\left[k_{x}^{2}+k_{y}^{2}-\mathbf{I} A-I_{z} B\right]} \\
Q_{x} & =Q_{x a}+k_{y} P_{z}, \\
Q_{y} & =Q_{y a}-k_{x} P_{z}, \\
Q_{z} & =Q_{z a}+k_{y} P_{x}-k_{x} P_{y},
\end{aligned}
$$

with $I_{i}=P_{i} P_{i}^{*}$ being $i=x, y, z, \kappa=\epsilon_{r}^{-1}$ and the " $a$ " field components are fully defined in. ${ }^{7-10,14}$

A nonlinear system of equations of the form $\mathbf{P}=\mathbf{U}(\mathbf{P})$ is made up by Eqs. (8)-(10). This system of equations can be solved by a fixed-point iterative process, in which we attempt to solve an process of the form $\mathbf{P}^{(p+1)}=\mathbf{U}\left(\mathbf{P}^{(p)}\right)$, where $p=0,1,2, \ldots$ denotes the iterations within the fixed-point procedure. We point out that the fixed-point process must be performed at each time step in the FDTD simulation. The scheme is based on an initial guess of $\mathbf{P}$, which we take to be equal to the (known) linear-domain quantity, after which the subsequent iterations are carried out improving the accuracy of the results at every iteration. Fig. 2 shows the convergence of the fixed-point iterative process. It can be seen that with 4 iterations the proper solution is achieved. Here, 15 iterations has been stablished in order to cover the worst convergence situations due to higher intensities or other issues. More details regarding the convergence condition for fixed-point iterative process and FDTD can be found in. ${ }^{13,17}$ 


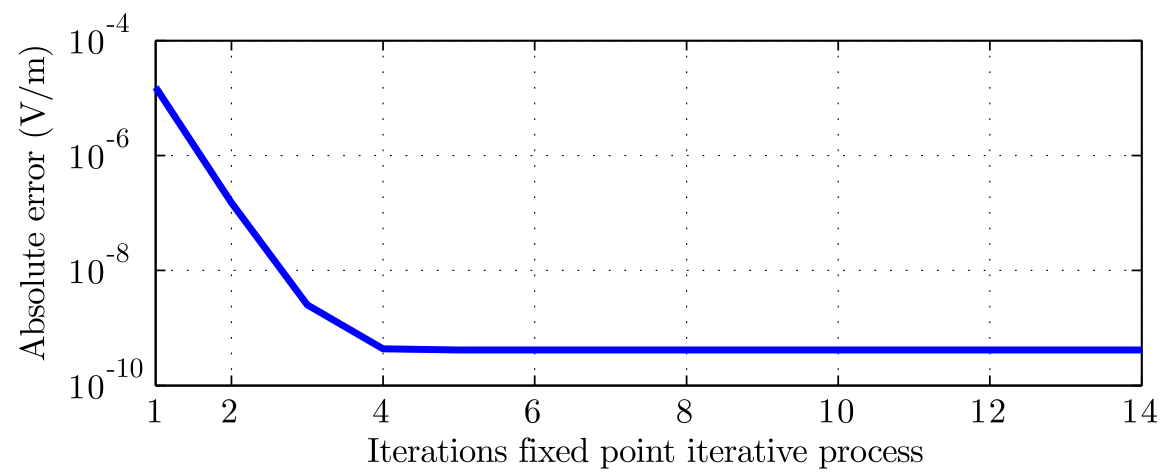

Figure 2. Convergence as a function of the number of iterations of the fixed-point iterative process.

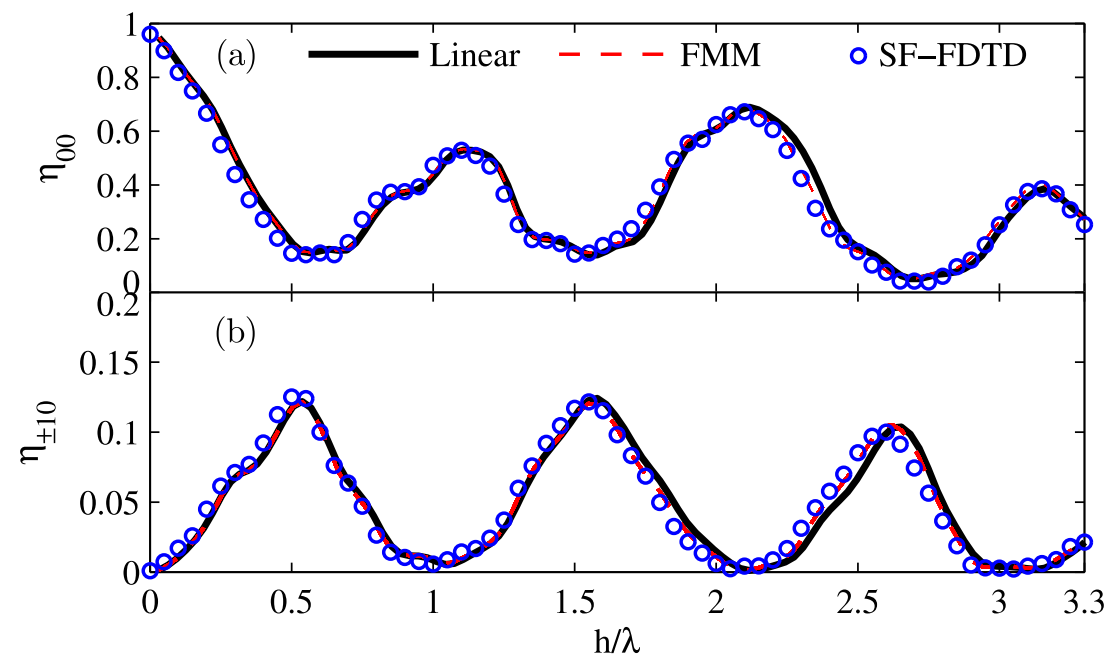

Figure 3. Diffraction efficiencies of a nonlinear binary grating computed by means of SF-FDTD and FMM. (a) Zeroth order. (b) First orders.

\section{RESULTS}

\subsection{Two-dimensional nonlinear binary grating}

We first apply the SF-FDTD presented here to the simulation of a two-dimensionally periodic nonlinear binary grating possessing a nonresonant nonlinearity in the material that fills the pillars (see Fig. 1b). The substrate is assumed to be $\mathrm{SiO}_{2}$ with $n_{s}=1.46$, and the pillar is filled with nonlinear $\mathrm{TiO}_{2}\left(n_{g}=2\right), A=10^{-19} \mathrm{~m}^{2} / \mathrm{V}^{2}$ and $B=2 A$. The period of the structure is $\Lambda=2.1 \lambda_{0}$. Figure 3 illustrates the results obtained with the method presented here along with those obtained with the FMM approach. ${ }^{15,18}$ The FDTD setup is based on a grid of $84 \times 84 \times 600$ cells in the $x, y$, and $z$ directions, respectively. The wavelength $\lambda_{0}$ is $633 \mathrm{~nm}$ and the spatial and time resolutions are $15.83 \mu \mathrm{m}$ and $1.76 \times 10^{-8} \mathrm{~ns}$. The input intensity is $10 \mathrm{GW} / \mathrm{cm}^{2}$. As can be seen in Fig. 3 both methods are consistent, thus validating the SF-FDTD method proposed here.

The FDTD setup for the results shown in Fig. 4 is based on a grid of $150 \times 150 \times 900$ cells. The wavelength is again $633 \mathrm{~nm}$, and the spatial and time resolutions are $10.56 \mu \mathrm{m}$ and $1.15 \times 10^{-8} \mathrm{~ns}$. Following the same strategy as for Fig. 3, the nonlinear effect is only included in the pillar (see Fig. 1b). Here, only the Kerr effect is considered, thus $\chi_{0}^{(3)}=A / 6=2.1 \times 10^{-20} \mathrm{~m}^{2} / \mathrm{V}^{2}$ and $B=0$. The substrate index is $n_{s}=n_{g}=1.5$, the TE illumination wavelength is $\lambda_{0}=1 \mu \mathrm{m}$, the period is $\Lambda=2.5 \lambda_{0}$, and the input intensity is $265.6 \mathrm{GW} / \mathrm{cm}^{2}$.

The results illustrated in Fig. 4a-b shows the diffraction efficiencies for the zeroth and the first orders as a function of the thickness of the nonlinear pillar with oblique angle of incidence. Shifting of the diffraction curves can be seen as the normalised thickness is increased. This phenomenon is due to the fact that the effective refractive index seen by the light is also increased as the amount of nonlinear material grows. ${ }^{13,14}$ Here a similar 


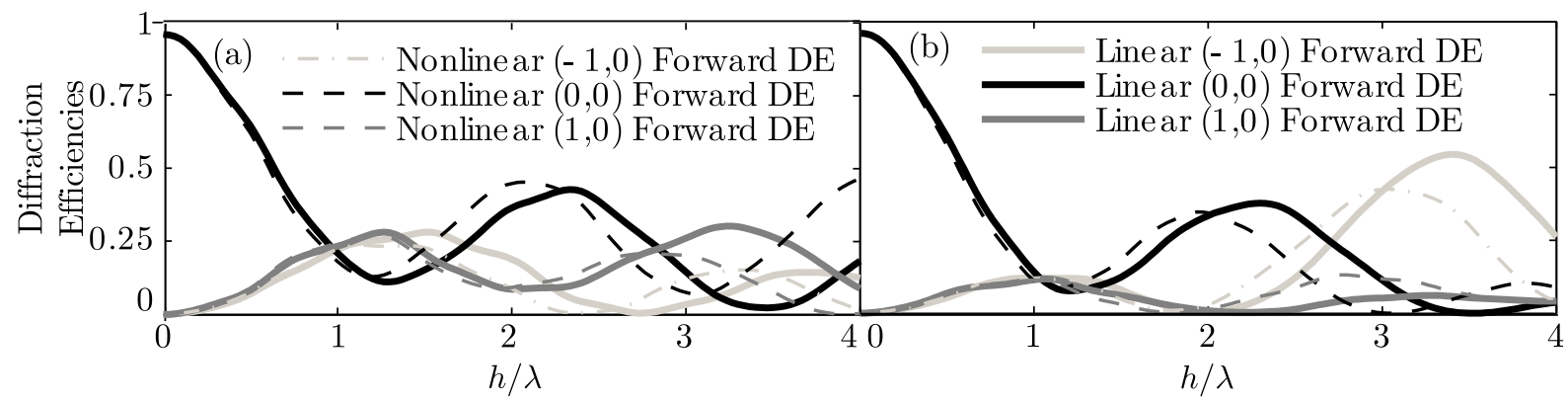

Figure 4. Diffraction efficiencies for 3D binary gratings nonlinear material filling the pillar. Linear and nonlinear cases are shown in order to show the shift produced in the curves due to the Kerr effect. Theta angle of incidence is $10^{\circ}$. (a) Rectangular pillars $\left(f_{x}=0.25\right.$ and $\left.f_{y}=0.75\right)$ and (b) binary grating $\left(f_{x}=f_{y}=0.5\right)$.

\begin{tabular}{cccccc}
$\Delta(\mathrm{nm})$ & $\Delta t(\mathrm{ps})$ & $x(\mathrm{~nm})$ & $y(\mathrm{~nm})$ & $z(\mathrm{~nm})$ & Time $(\mathrm{ps})$ \\
\hline 156.25 & $1.72 \cdot 10^{-6}$ & 375 & 9.38 & 1562.5 & 0.21 \\
\hline \hline
\end{tabular}

behaviour as above (see Fig. 3) can be identified but a more complex interaction of the nonlinear media is seen due to the effective increase of the optical length of the nonlinear pillar with oblique angle of incidence.

\subsection{One-dimensional nonlinear coated binary grating}

In order to show the potential of the SF-FDTD method here presented, a one-dimensially nonlinear coated binary grating $(\mathrm{CBG})$ is considered. A cross-section of the CBG is shown in Fig. 1c. This device can be assumed as a one waveguide layer and one diffraction grating. This setup is the basis of resonant waveguide grating (RWG) and one of the most important characteristic of RWG is that reflectance can change rapidily from near to 0 to $100 \%$ as a function of the input field parameters: wavelegth, angle of incidence and polarization. In this work, a subwavelength structure is considered and also a nonlinear susceptibility is considered in the $\mathrm{TiO}_{2}$ layer. The parameters of the structres are: grating material $\mathrm{SiO}_{2}\left(n_{3}=1.47\right), \Lambda_{x}=375 \mathrm{~nm}, f_{x}=0.5, d_{1}=300 \mathrm{~nm}$, coating $\mathrm{TiO}_{2}\left(n_{2}=2\right), d_{2}=200 \mathrm{~nm}$, and the thickness on the bottom of the grating $\left(d_{3}\right)$ and also in the sidewalls is 50 $\mathrm{nm} .{ }^{19}$ It is worth to note that even being this case a one-dimensially periodic device the 3D SF-FDTD is here considered with a non-variant $z$-axis. Since FDTD is based on updating the electromagnetic field as a function of space and time, a steady-state for RWG must be ensured. The setup for the FDTD engine is summarised in Table 1.

(a)

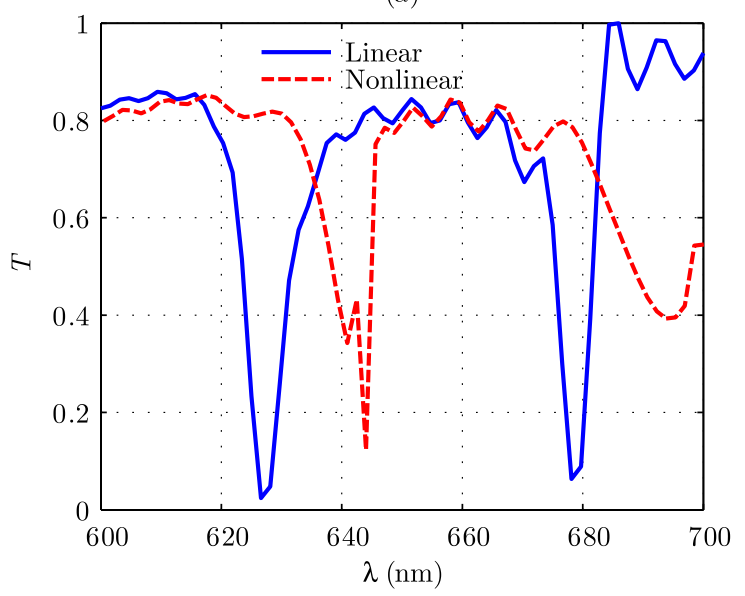

(b)

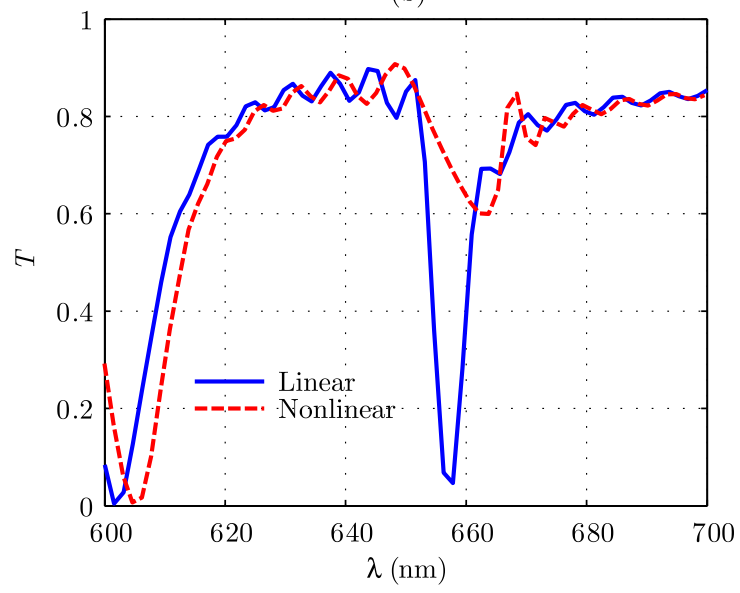

Figure 5. Transmission of a RWG as a function of the wavelegth in linear and nonlinear regime. In both cases $A=$ $1.47 \cdot 10^{-20}(\mathrm{~m} / \mathrm{V})^{2}, B=2 A$ and input intensity of $100 \mathrm{GW} / \mathrm{cm}^{2}$ : (a) TE polarization. (b) TM polarization. 


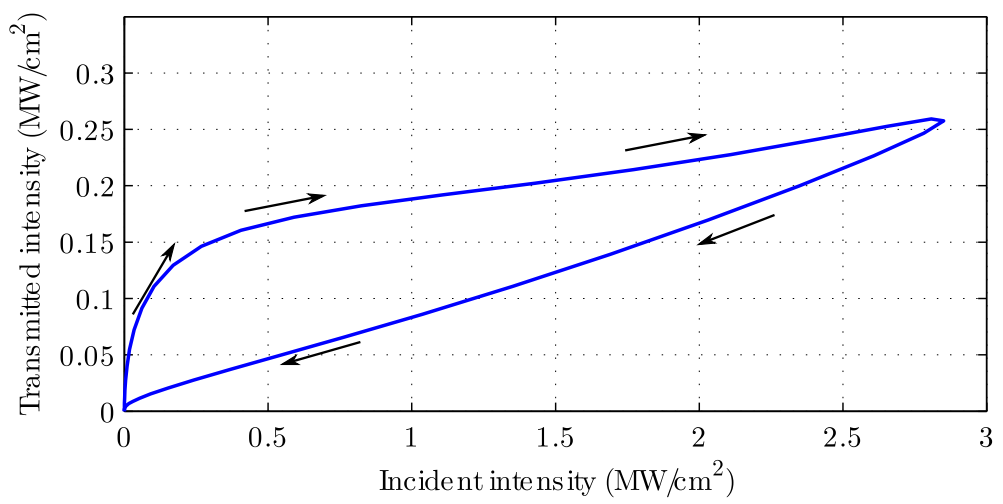

Figure 6. Intensity of transmitted light as a function of intensity of incident light for $\lambda=625 \mathrm{~nm} \cdot \chi_{0}^{(3)}=1.47 \cdot 10^{-14}$ $(\mathrm{m} / \mathrm{V})^{2}$.

Fig. 5 shows the transmission of the CBG as a function of the wavelength for the linear and nonlinar case. It is worth to note that here nonresonant electronic response $(A=2 B)$ as physical mechanism for modelling the nonlinear refractive index ${ }^{1}$ is assumed. The results show a shifting in the transmission curves in nonlinear regime. Fig. 5a shows how triggering the nonlinear behaviour of the $\mathrm{TiO}_{2}$ layer the sharp resonances can be modified in terms of wavelegth. For the TM case the reduction of the resonance close to $657 \mathrm{~nm}$ is almost reduced in the nonlinear case.

One of the most important features of FDTD is the possibility of analyse behaviour of optical devices as a function of time. Fig. 6 shows the transmitted intensity calculated by the nonlinear SF-FDTD method as a function of the incident light with $625 \mathrm{~nm}$ wavelegth. Here, only the Kerr effect is considered, thus $A=\chi_{0}^{(3)}$ and $B=0$. The transmitted intensity has an obvious bistable phenomenon.

It is worth to note that as it can be seen in Table 1 the time window considered is huge compared to the time resolution of the SF-FDTD method. In RWG the steady-state is achieved after many time-steps, thus the results here included have been obtained ensuring that the RWG has reached the steady-state and that the output intensity remains constant. More precisely, for the results shown in Fig. 5, 120.106 time steps have been considered. Regarding, the results shown in Fig. 6 the same procedure has been followed. In this case instead of an harmonic illumination, a bandpass Gaussian pulse centered at $625 \mathrm{~nm}$ has been considered. In this case even a higher number of time steps has been considered in order to ensure the steady-state in the RWG. With this setup, acceleration strategies have been considered in order to reduce the time simulation costs. In order to achieve this reduction parallel strategies have been considered. ${ }^{11,20,21}$ Basically, the auto-vectorisation performed by the compiler and OpenMP have been considered. For enabling properly the auto-vectorisation an efficient memory alignment, a correct loop count and proper structures were considered.

In order to analyse the computational performance of the SF-FDTD with the different architectures available, a set of grids has been defined:

- $\mathrm{SMALL}[40 \times 40 \times 300], n_{\text {step }}=3026, \mathrm{~S}^{\mathrm{L}}=92 \mathrm{MB}, \mathrm{S}^{\mathrm{NL}}=131 \mathrm{MB}$.

- $\operatorname{MEDIUM}[180 \times 60 \times 400], n_{\text {step }}=4386, \mathrm{~S}^{\mathrm{L}}=829 \mathrm{MB}, \mathrm{S}^{\mathrm{NL}}=1175 \mathrm{MB}$.

- $\mathrm{LARGE}[300 \times 80 \times 500], n_{\mathrm{step}}=5830, \mathrm{~S}^{\mathrm{L}}=2304 \mathrm{MB}, \mathrm{S}^{\mathrm{NL}}=3264 \mathrm{MB}$.

The results achieved by the auto-vectorisation provides a considerable reduction of the time costs due to the proper memory alignment and the correct implementation of the loops. In order to clarify this issue the Table 2 shows a summary of the results provided by the auto-vectorisation for the three simulation sizes. The version compiled with the flag -00 is the sequential code without any auto-vectorisation. The sequential version with auto-vectorisation is considered here as the best sequential code, due to the fact that it is the best option achievable by an standard programmer without advanced knowledge in computer optimisation. Finally, the degree of improvement between these two versions is also summarised. It can be seen that with auto-vectorisation the 


\begin{tabular}{|c|c|c|c|}
\hline Flag & $\mathrm{T}_{\text {SMALL }}$ & $\mathrm{T}_{\text {MEDIUM }}$ & $T_{\text {LARGE }}$ \\
\hline $2-00$ & $22^{\prime \prime 2} 7^{\prime \prime}$ & "2h49'17" & "10h7'14" \\
\hline Seq $(-03)$ & $5^{\prime} 27^{\prime \prime}$ & $43^{\prime} 18^{\prime \prime}$ & 2h32'2" \\
\hline Ratio & 3.89 & 3.91 & 4.99 \\
\hline
\end{tabular}

Table 2. Time cost analysis of the auto-vectorisation introduced by the compiler.

\begin{tabular}{llll}
\hline Platform & SMALL & MEDIUM & LARGE \\
\hline \hline GTX 670 & 5.41 & 5.72 & 5.39 \\
GTX Titan X & 14.73 & 14.39 & 14.38
\end{tabular}

Table 3. Relative speedup considering as sequential code the CPU code with OpenMP.

application runs five times faster, thus validating the implementation of the sequential version here implemented and the proper usage of the auto-vectorisation options of the compiler.

Finally, Table 3 shows a summary of the results with the relative speedup. It is defined as the relationship between the time simulation cost of the parallel version and the best CPU version. Here, the best CPU version with auto-vectorisation and OpenMP is considered as reference in order to compare the different platforms accurately. This analysis provides a rigorous metric of the performance of the different architectures and programming paradigms considered since GPU is compared with the performance provided by a single CPU processor with auto-vectorisation. The results reveal that GPU computing is more than 5 times faster for a single GTX 670 and close to 15 for the GPU Titan X.

\section{CONCLUSIONS}

Concluding, we introduced the SF-FDTD for the analysis of 2D periodic structures with nonlinear materials. A fixed-point iterative process has been included in order to solve the non-linear system of equations that is established due to considering the vectorial definition of the third-order nonlinear susceptibility in three dimensions. The reliability of the method is demonstrated by means of the analysis of dielectric binary gratings with both SF-FDTD and a recent nonlinear implementation of the FMM. Afterwards, SF-FDTD method is applied to the analysis of two-dimensionally binary gratings with different fill factors. In both cases the results are consistent with literature and tuneable optical structures are obtained, since a shift in the diffraction curves are obtained when the nonlinear effect is triggered in the structures. The method has been also applied to nonlinear resonant waveguide gratings in two dimensions. For nonlinear RWG the shifting in the resonance peaks and the bistability of nonlinear effects has been also analysed. The bistability curve also demonstrates the potential of finite-difference time-domain schemes in which the time transient behaviour of the optical device can be easily analysed.

The relative speed up is computed considering as reference the best code version on CPU (with autovectorisation and OpenMP). The results show that GPU computing provides the best results reducing in more than 14 times the simulation time for all scenarios here considered (compared with the best CPU version with OpenMP directives).

Concluding, here an extension of the split-field finite-difference time-domain method has been detailed for the analysis of materials with a vectorial nonlinear susceptibility in two-dimensially periodic structures. This feature has not been developed to the best of our knowledge in the SF-FDTD. Currently, the authors are working on including more parallel strategies in this formulation in order to reduce the time and resources needed for updating the electromagnetic field components as a function of time.

\section{ACKNOWLEDGMENTS}

This work was supported by the Ministerio de Economa y Competitividad of Spain under project FIS2014-56100C2-1-P and by the Generalitat Valenciana of Spain under projects PROMETEOII/2015/015, ISIC/2012/013 and $\mathrm{GV} / 2014 / 076$. 


\section{REFERENCES}

[1] Boyd, R. W., [Nonlinear Optics], Academic Press, 3 ed. (2008).

[2] Flytzanis, C. and Oudar, J. L., [Nonlinear Optics: Materials and Devices], Springer-Verlag Berlin Heidelberg (1986).

[3] Soljačić, M., Luo, C., Joannopoulos, J. D., and Fan, S., "Nonlinear photonic crystal microdevices for optical integration," Opt. Lett. 28, 637-639 (Apr 2003).

[4] Yee, K., "Numerical solution of initial boundary value problems involving maxwell's equations in isotropic media," Antennas and Propagation, IEEE Transactions on 14, 302-307 (May 1966).

[5] Taflove, A., Oskooi, A., and Johnson, S. G., eds., [Advances in FDTD Computational Electrodynamics: Photonics and Nanotechnology], Artech House (2013).

[6] Roden, J., Gedney, S., Kesler, M., Maloney, J., and Harms, P., "Time-domain analysis of periodic structures at oblique incidence: orthogonal and nonorthogonal fdtd implementations," Microwave Theory and Techniques, IEEE Transactions on 46, 420-427 (Apr 1998).

[7] Oh, C. and Escuti, M. J., "Time-domain analysis of periodic anisotropic media at oblique incidence:an efficient fdtd implementation," Opt. Express 14, 11870-11884 (Nov 2006).

[8] Miskiewicz, M. N., Bowen, P. T., and Escuti, M. J., "Efficient 3d fdtd analysis of arbitrary birefringent and dichroic media with obliquely incident sources," Proc. SPIE 8255, 82550W-82550W-10 (2012).

[9] Shahmansouri, A. and Rashidian, B., "Comprehensive three-dimensional split-field finite-difference timedomain method for analysis of periodic plasmonic nanostructures: near- and far-field formulation," J. Opt. Soc. Am. B 28, 2690-2700 (Nov 2011).

[10] Shahmansouri, A. and Rashidian, B., "GPU implementation of split-field finite-difference time-domain method for Drude-Lorentz dispersive media," Prog. Electromagn. Res. 125, 55-77 (2012).

[11] Francés, J., Bleda, S., Álvarez, M. L., Martínez, F. J., Márquez, A., Neipp, C., and Beléndez, A., "Acceleration of split-field finite difference time-domain method for anisotropic media by means of graphics processing unit computing," Optical Engineering 53(1), 011005-011005 (2013).

[12] Joseph, R. and Taflove, A., "FDTD Maxwell's equations models for nonlinear electrodynamics and optics," Antennas and Propagation, IEEE Transactions on 45, 364-374 (Mar 1997).

[13] Francés, J., Tervo, J., and Neipp, C., "Split-field finite-difference time-domain scheme for Kerr-type nonlinear periodic media," Prog. Electromagn. Res. 134, 559-579 (2013).

[14] Francés, J., Tervo, J., and Márquez, A., "Tensorial split-field finite-difference time-domain approach for second- and third-order nonlinear materials," J. Opt. Soc. Am. B 30, 1711-1719 (Jun 2013).

[15] Bej, S., Tervo, J., Svirko, Y. P., and Turunen, J., "Modeling the optical kerr effect in periodic structures by the linear fourier modal method," J. Opt. Soc. Am. B 31, 2371-2378 (Oct 2014).

[16] Berenger, J.-P., "A perfectly matched layer for the absorption of electromagnetic waves," Journal of Computational Physics 114(2), 185 - 200 (1994).

[17] Ammann, M., Non-Trivial Materials in EM-FDTD, Master's thesis, Department of Physics, Swiss Federal Institute of Technology (2007).

[18] Bej, S., Tervo, J., and Svirko, Y. P., "Fourier modal method for crossed gratings with kerr-type nonlinearity," Proc. SPIE 9131, Optical Modelling and Design III 913116, 913116-913116-9 (2014).

[19] Karvinen, P., Applications of resonant waveguide gratings, PhD thesis, Department of Physics and Mathmatics, University of Eastern Finland (2010).

[20] Francés, J., Bleda, S., Márquez, A., Neipp, C., Gallego, S., Otero, B., and Beléndez, A., "Performance analysis of SSE and AVX instructions in multi-core CPUs and GPU computing on FDTD scheme for solid and fluid vibration problems," The Journal of Supercomputing 70(2), 514-526 (2014).

[21] Francés, J., Otero, B., Bleda, S., Gallego, S., Neipp, C., Márquez, A., and Beléndez", A., "Multi-GPU and multi-CPU accelerated FDTD scheme for vibroacoustic applications," Computer Physics Communications 191, 43 - 51 (2015). 